\title{
Procedure for measuring solar and visible properties simultaneously of glazing with complex internal or external structures
}

\author{
A.R. Gentle and G.B. Smith \\ School of Physics and Advanced Materials; University of Technology, Sydney \\ Po Box 123, Broadway, NSW 2007 Australia \\ *Corresponding author: g.smith@uts.edu.au
}

Received Month X, XXXX; revised Month X, XXXX; accepted Month X, XXXX; posted Month X, XXXX (Doc. ID XXXXX); published Month X, XXXX

\begin{abstract}
Accurate solar and visual transmittance of materials in which surfaces or internal structures are complex are often not easily amenable to standard procedures with laboratory based spectrophotometers and integrating spheres. Localized "hot spots" of intensity are common in such materials, so data on small samples is unreliable. A novel device and simple protocols have been developed and undergone validation testing. Simultaneous solar and visible transmittance and reflectance data has been acquired for skylight components and multilayer polycarbonate roof panels. The pyranometer and lux sensor set-ups also directly yield "light coolness" in Lumens/Watt. Sample areas must be large and though mainly in sheet form some testing has been done on curved panels. The instrument, its operation, and the simple calculations used are described. Results on a sub-set of diffuse and partially diffuse materials with no hot-spots have been cross-checked using $150 \mathrm{~mm}$ integrating spheres with spectrophotometer and the Air Mass 1.5 spectrum. Indications are that results are as good or better than with such spheres for transmittance but reflectance techniques need refinement for some sample types.

OCIS codes: (350.4880) Optical standards and testing; (350.6050) Solar energy; (220.2945) Illumination design;

(290.5880) Scattering rough surfaces.

http://dx.doi/org/10.1364/AO.99.09999;(290.5880)
\end{abstract}

\section{Introduction}

The energy and daylighting performance of skylights, windows and roof glazing systems is now regarded as an essential element in modeling and assessing the thermal performance of buildings, and the quality and amount of daylight supplied. While transmittance is the key issue, roof solar reflectance is also important for keeping these elements cool and helping to raise average urban albedo [1-3]. Absorbed solar energy and emittance are also needed for determining the final U-value and Solar Heat Gain Co-efficient (SHGC), and hence for modeling the net solar heat gain and lighting input of a complete skylight design including well or tube walls and any light diffusing elements usually placed at ceiling level. A skylight design cannot be reliably rated until all of these steps are completed and input data is sufficiently accurate.

External visible reflectance also impacts on building and suburb aesthetics, and on glare management. Basic radiant parameters were the focus in this initial study namely normalhemispherical solar transmittance and reflectance (Th,sol and $\mathrm{Rh}, \mathrm{sol}$ ), normal-hemispherical visible transmittance and reflectance (Th,vis and Rh,vis), and thermal emittance $\mathrm{E}$ of surfaces. This technique represents a novel approach to finding the four parameters Th,sol, Rh,sol, Th,vis, Rh,vis, along with daylight "coolness" via lumens per watt transmitted. Since the source of light used is the sun, lighting related parameters may differ from some standard results for the same diffusing materials based on their use with particular standard lamps with different spectral output to the sun. Both types of data are needed as some of these complex materials are used in both lamp housings and in skylights and roof glazing.

Light transmitting products today often need verifiable "star", "energy" or "light output" ratings and in many places are subject to building code limitations. This makes these four optical parameters, and standard techniques for their measurement, essential knowledge for suppliers, and the data should be available to consumers, builders and architects. Standards for their determination for light transmitting materials which have smooth surfaces with no internal structure, whether specular or uniformly diffuse, have been established for example in ASTM E903-96 [4]. Others for reflectance, are not directly useful for transmittance, and require very large flat opaque areas for use with pyranometers such as E1918 [5], for daylighting with a photometer E972 [6], and for solar transmittance using a pyranometer E1084 [7] which uses an enclosure to integrate transmitted radiation. While of use in the field to some extent but often awkward there is no simple analogue to reflectance using E903-96 which can be applied easily to a wide range of complex materials before installation as is currently done with spectrophotometers plus small integrating spheres. When 
available and reliable the latter's data can be used in established models for basic multi-layer window systems as in normal double and triple glazing which has no transverse walls bridging gaps, or other inserts. Multi-wall polymer extruded panels however have internal walls which do bridge and support the main layers. These complicate the optics and generate "hot" zones in the emergent radiant fields. They can be studied ex-situ by our method.

Most of the standards cited above mainly describe the technique itself, and sometimes extend to in situ data on installed systems. Our ultimate aim is different. It is to provide the basis for a universal approach to a standardized complex transmitting materials data-base analogous to that used for noncomplex glazing products (e.g The International Glazing Data Base -IGDB) [8]. Assuming very large integrating spheres of order 4 to 5 meters in diameter do not exist in most laboratories a simple, easily duplicated technique is needed, with an ability to characterize uniformly illuminated large areas. That is our goal. A possible alternative for moderate sized spheres is the recent spectrophotometric - integrating sphere technique described by Nilsson et al [9] which has one key aspect similar to ours, namely the use of a special secondary panel to diffuse the sample transmitted beam. Their focus was on avoiding losses when some transmitted light strikes the sphere port edges and also because in general integrating spheres work better when light striking their walls is less specular. Edge losses are of no concern in our size samples. Further discussion on similarities and differences between both techniques follows later. A standard beam profile falling on the detector for any sample is a feature of our transmittance technique. Its solar and visible results are direct. That is there is no need to first acquire a spectrum, though a spectral study is possible.

Thus a variety of glazing elements cannot be characterized with confidence using the techniques outlined in the current standards because of complexities in the way they transmit or reflect radiation. Complex computer algorithms such as used in WINDOW 7.1, 7.2 [10] can compute results for some complex glazing systems, including those with multiple scattering elements and multilayers, such as blinds and cellular shades. The scattering profile of each element given by its Bi-directional transmittance distribution and Bi-directional reflectance distribution functions (BTDF and BRDF respectively) must be known accurately via experiment or via ray tracing. However approximations and data used mean some experimental data on each element and final validation are needed for model input and assessment of final accuracy. Thus the full experimental capability outlined here is rarely available to date for complete structures and some component layers. Polymer products with surface structure may have small unavoidable production defects, which can play a significant role in complicating output patterns and the development of "hot spots". Even ideal feature shapes can cause problems. For example where sharp direction changes arise in surface contours Haas-Goochen shifts in total internal reflectance cause complex interference patterns [11].

Another issue often missed is the asymmetry in both transmittance and reflectance that can arise when one side is smooth and the opposite contoured as shown in fig. 1 . This does not occur for transmittance in normal optical systems when both sides are optically smooth. The origins are that total internal reflection (TIR) regimes are different for each side. For example for normal internal incidence, on the smooth side TIR is irrelevant but can be large on parts of the contoured side. Reflectance asymmetry is common however as it occurs even if all surfaces are smooth with opposite sides having different

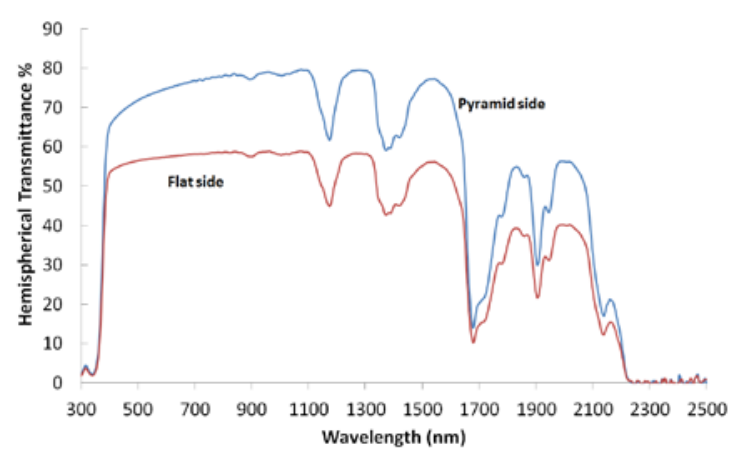

Fig. 1. Asymmetry in spectral transmittance with structure on one side only of a PMMA panel. Incident side labeled.

coatings, for example if one only has a solar reflecting, low e coating. In some complex materials of interest in this work both contour and coating differences may be present on opposite sides. Our instrument can also cope with this interesting and important case as we always measure all four parameters for both up and down panel orientations.

\section{Materials of interest}

The prime motivation for our study was a need to better characterize the energy and lighting properties of sheets with prismatic structures on one side only. Examples appear in fig. 2, where "hot spots" or bright zones are clearly visible.

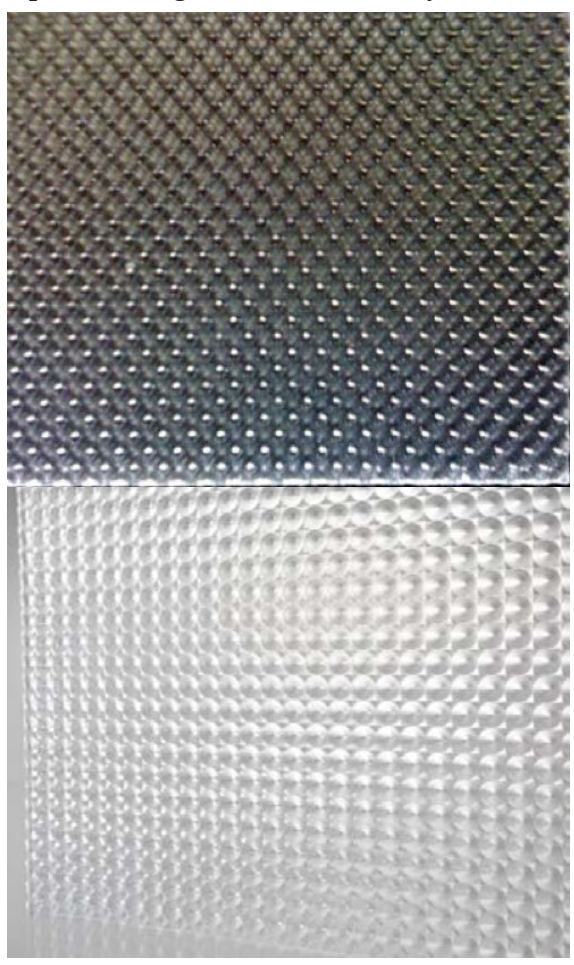

Fig. 2. Illuminated acrylic sheets showing the contoured side and "hot" zones in the transmitted visual field. 
Other very bright domain structures are possible apart from the intense localized bright spots seen in fig. 2. In multi-wall sheeting of the types shown in fig. 3, the bright zones can be elongated and cause severe discomfort glare. Actual bright zone structures for solar and visible radiation, and hence transmission intensity profiles, depend on both direction of incidence and the sensing position. This is formally given by the bi-conical or directdirect transmittance, or the BRDF for multiple input and output ray directions. Much data is needed on complex glazing if these parameters are to be used to find the four parameters we require. In contrast our technique for each panel orientation needs just four fast measurements for fixed solar irradiance and illuminance. For existing total solar energy and lumens transmittance it is usual to use the direct-hemispherical transmittance, which this instrument when used as described, also gives directly. That means as noted above that we have to measure the sum, or a fixed fraction thereof, of radiation transmitted in all directions. We confine the incident beam to be normal to the sample to provide a common basis for product comparison as is done with normal glazing. Variable incidence directions and the impact of hemispherical and partially diffuse solar radiation are for future study but can be easily done with this instrument.

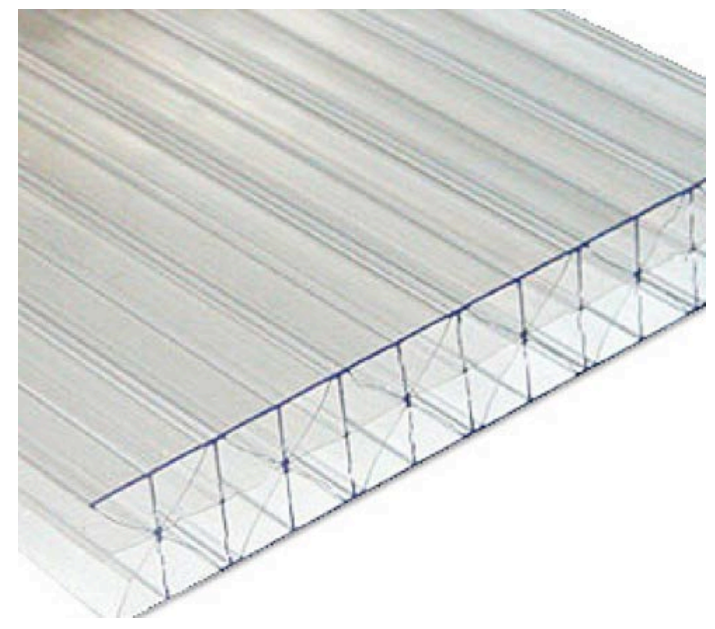

Fig. 3. An extruded multi-wall clear wall polycarbonate roofing panel. Doping of panel walls is often done, and such panels can also be studied in this rig.

It was also considered an important goal if this technique was to be suitable as a standard procedure which was not time consuming, to locate the transmittance sensor in a fixed position in relation to the sample surface. A hierarchy of experimental restraints follows (1) Large sample and beam sizes are needed to correctly average impacts of surface structure variations on total transmitted radiation (2) The sensor must sample a common representative sum or fraction of all transmitted radiation (3) An additional element between sample and sensor is needed to homogenize the output radiance or luminance field (4) For good signal levels the homogenizer must combine high transmittance with either uniform output or a fixed radiance output map for any incidence profile. The process of homogenization is central to the transmittance approach outlined here. It is achieved by insertion of a standardized and suitable diffusing panel directly below the sample. The extent to which this panel meets important restraint (4), namely a uniformly diffuse output beam for solar radiation incident on any sample of interest plus a high enough transmittance for signal levels from the combination of sample and integrator to be sufficiently high for good accuracy, can be tested in practice using projected intensity maps on a screen or via rigorous studies of BTDF for the homogenizer. If it is near Lambertian it should be suitable. The polymer material used approached this ideality with good uniformity of transmittance when behind all studied samples and with normal-hemispherical transmittance of $88 \%$ to $89 \%$ as measured with an integrating sphere spectrophotometer. Incidentally such a standard diffuser material itself is very useful for energy efficient, quality skylights by providing uniform lighting over a room floor while minimizing area needed for desired lumens and lux, and is also of high interest for luminaires for maximizing use of lamp output.

Sample total thickness is important when dealing with complex polymers and diffuse polymers in general. Thickness can impact on the diffuse component as a function of wavelength, which can have an impact on results from some instruments. For example thin sub-mm films of PTFE can have a significant normal component in T or R in the NIR while being totally diffuse below $850 \mathrm{~nm}$. Examples of this include solar cell encapsulant polymers [12]. Here such spectral shifts in behavior are only an issue for reflectance since our instrument uses an excellent secondary diffuser for transmittance studies. Thicknesses in polymer sheets used in this study ranged from $3 \mathrm{~mm}$ to $4 \mathrm{~mm}$, including where applicable the distance from the flat side to the top of surface pyramids whose apex height is typically $1 \mathrm{~mm}$.

In use, especially on roofs and skylight ceiling diffusers, these panels with complex surfaces can be formed into a curve, so it is useful to be able to sample transmittance of a complete curved element which is impossible with most standard techniques but not for transmittance in this technique. We have carried out such studies in this rig but comment only here and confine this introductory paper's details to complex flat panels.

Other systems where this technique may have value include

(1) Double and triple glazing whose internal space includes solid meshes, for example a metal mesh or grid made from twisted or planar metal rods or wires. Panel orientation and metal twist details enable angular selectivity of three quantities, transmittance, reflectance and external field-of-view.

(2) The combination of normal glazing or skylights with blinds and curtain materials

(3) Roof and wall glazing which combine solar cells and provision of daylight

(4) Diffuse wall sections

(5) Many windows or transmitting roof fittings with inscribed patterns, lettering or art-work.

This instrument could also be adapted for reflectance studies on diffuse opaque materials with complex surface structures. A technique previously developed for in-situ roofing studies [13] and as a proposed alternative for complex opaque surfaces to the E1918 standard, namely "E1918A", provided some ideas for this instrument. Our procedure for reflectance has some differences to "E1918A" due to a focus on transmitting materials. An "E1084B" emerging from this work would be a possible analogue for transmittance only. Further studies are needed, for example to see if sample specific modifications and rig orientation away from 
the normal to sun improves solar or visible reflectance data as a basis an "E1084B" standard, as an extension of "E1918A" for complex materials which aren't opaque. Most samples of interest in this initial study have $\mathrm{R}<10 \%$ and are diffuse so for them results are close to what is expected. "E1918A" was for in-situ studies on a given building's opaque roof. In this rig fixed orientation with respect to incoming solar radiation is ensured, which is better suited to compare products, surfaces and roofing "mock-ups" in a standardized fashion. Solar radiation intensity in our case is not fixed at any particular air-mass such as 1.5 (AM1.5) and depends on location, time of day and season. Indoor lamp arrays could be used but add much cost. Cross-checks were used on our own laboratory based spectral results on suitable diffuse samples with no hot spots, using AM1.5 spectra. They agree well with rig results. Total solar irradiance during all data collection reported here lay between 1,000 and $1,100 \mathrm{Wm}^{-2}$ with most between 1,000 and $1,040 \mathrm{Wm}^{-2}$ and with very low diffuse content. These characteristics may have ensured good compatibility with AM1.5 conditions.

\section{(3) Experimental design}

Images of the mobile rig from different viewing directions. and a scale drawing follow. A hollow square panel $25 \mathrm{~mm}$ thick enclosed by aluminum sheet contains the large sample aperture and supports the thin detector mounting arm as seen under development and in profile in fig. 4 prior to painting black. It is supported by an aluminum A-frame mounted on axially rotatable wheels. The solar radiation detector's thermal sensor mounting point is stood-off from the sample by $410 \mathrm{~mm}$. A side view schematic with key dimensions follows in fig. 5. The axial movement of the wheels plus panel tilt adjustments about the horizontal axis are adjusted regularly to ensure the support panel is always normal to the direct solar beam during data collection. Rotational adjustments about the vertical set alignment with the solar azimuth. Thus is checked by the perfect alignment of the two shadows from the two A-Frame support

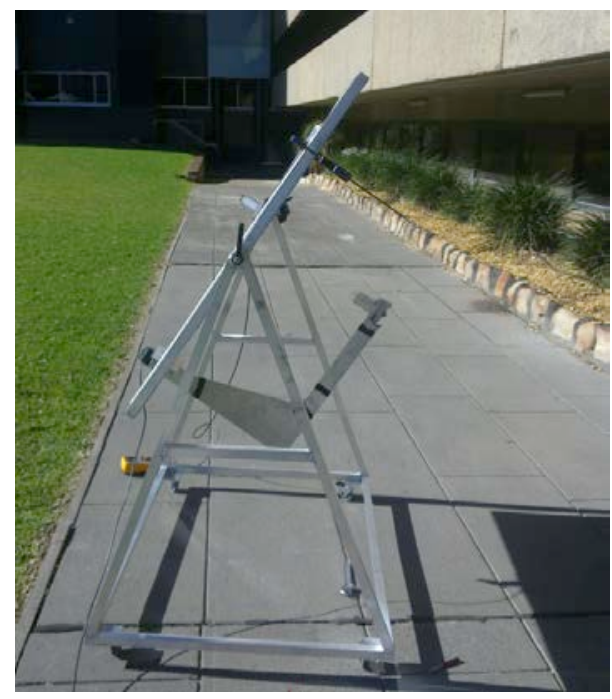

Fig. 4. Side-view of the rig under development (before painting back) in transmittance mode.

rods, which can be seen meeting on each side of the sample support panel. Slight misalignment is easy to discern. The tilt adjustment can then be set to maximize solar intensity normal to the sample. An ongoing check on both settings is the lack of any shadow from the reference pyranometer's support cylinder, along with any significant drift in the signal from this sensor.

Uniform Solar Radiation

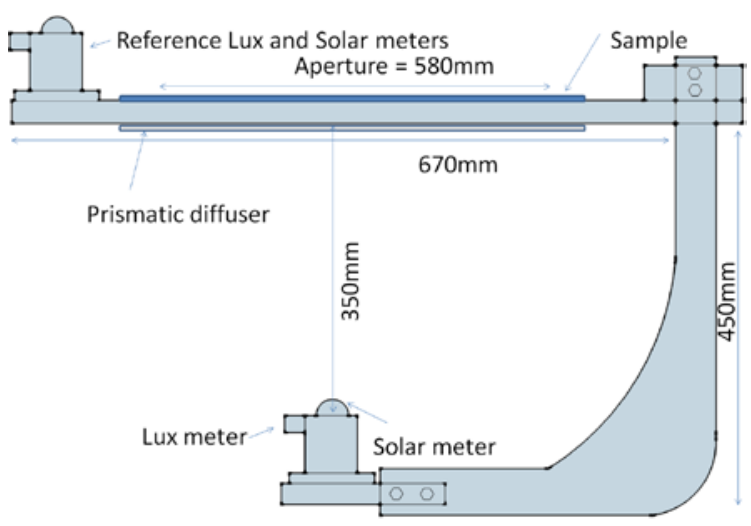

Fig. 5. Section-view schematic in the plane containing the sensor support arm, of the set-up for transmittance mode measurements. The sample aperture is $580 \times 580 \mathrm{~mm}$. Sample panel sides range from 610 to $660 \mathrm{~mm}$. The aluminum beam supporting the detector is $5 \mathrm{~mm}$ thick.

In fig. 5. the solar radiation sensor is positioned behind the midpoint of the sample-diffuser pair in transmittance mode. The parallel and identical reference sensors provide input solar intensity and daylight lux levels. The pyranometer thermopile surface is $60 \mathrm{~mm}$ above its mounting plate, $350 \mathrm{~mm}$ from the standard diffuser's back surface, and $375 \mathrm{~mm}$ from the rear surface of the sample of interest. Hence the thermopile is $(375+$ t) $\mathrm{mm}$ from the sample's front surface, with $\mathrm{t}$ sample thickness (typically ranging from $3 \mathrm{~mm}$ to $25 \mathrm{~mm}$ ). The smaller area lux photo-sensor is strap-mounted on one side of the pyranometer body and hence offset slightly from the normal to the sample mid-point by the sum of pyranometer radius of $20 \mathrm{~mm}$ and its own radius of $8 \mathrm{~mm}$. In reflectance mode the $350 \mathrm{~mm}$ shown in fig. 5 becomes the spacing to the sample front surface and the $375 \mathrm{~mm}$ the spacing to a pre-calibrated black rear surface. This is used as explained later, as a standard background component.

Fig.6 illustrates schematically how the optical responses of the sample and reference diffuser to incident sunlight are combined to produce a uniformly diffuse exit beam. With the sample plate removed the observed diffuse intensity would be in effect the $100 \%$ reference signal for transmittance if there was no background signal. A small correction to both this intensity and that when the sample plate is back in place is however required. This involves an additional measurement to find the background contribution to these signals. This comes mainly from secondary reflection off the black rear of the mounting plate, with a little from reflected radiation in the oblique field of view. 


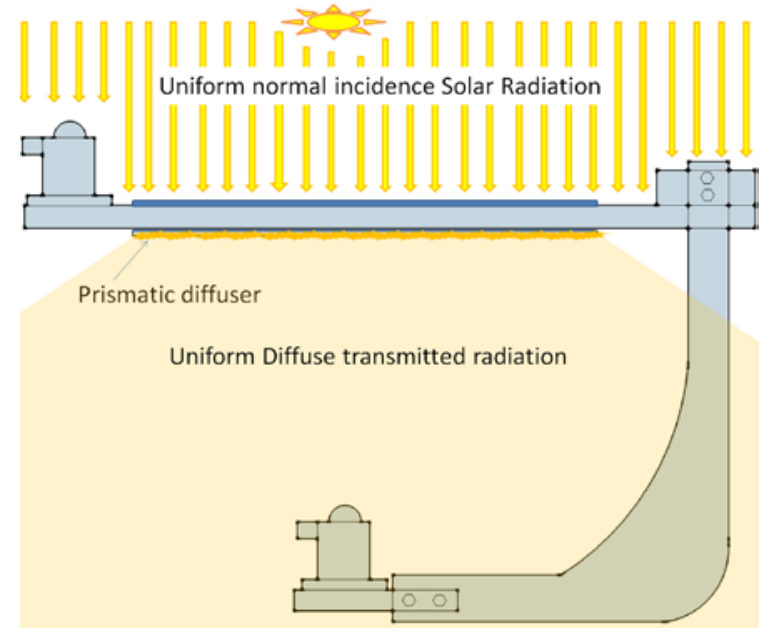

Fig.6. Cross-section schematic of the incident solar beam and the emerging radiant field, after passing through the sample panel and the special diffuser panel. The diffuser's role as shown, is to homogenize all radiation emerging from the sample plate.

The tilt system also enables a rotation though $180^{\circ}$ about the horizontal to switch from transmittance to reflectance modes. When the latter occurs the direct solar and light sensors must also be re-mounted on the new sun facing side but the sample sensors do not have to be moved. In future studies of reflectance a slightly oblique solar incidence to minimize shadowing impacts from the sample sensor onto the sample needs studying. A slight axial rotation of the whole rig can provide such a set up. But in these initial tests the sample sensor was mounted with its axis aligned to the sample normal to assess direct shadowing influence for different sample types. This shadowing was expected to become more important as the degree of specularity in the sample reflectance increases. A close-up of one sensor pair measuring direct solar flux and illuminance is in fig. 7 along with a back view of the sample sensor in reflectance mode showing its shadow. "Sensor pair" refers to the dual side-by-side detectors, one for solar energy intensity which uses a Middleton SK08-E pyranometer, and one for illuminance or lux which uses a Minolta light sensor head as seen linked by cable to a light meter. This pyranometer was chosen because of its narrower profile so as to minimize shadow area in reflection mode and back reflection from pyranometers with cooling wings. SK08-E meets ISO 9060 standards for a first class instrument. It has output sensitivity $1 \mathrm{mV}$ per $\mathrm{Wm}^{-2}$. At $75 \mathrm{~mm}$ in diameter the SK08-E base plate is the largest source of sample shadowing in reflectance mode. The round base plate is $410 \mathrm{~mm}$ from the sample's front surface.

For this initial study a normal sensor mounting was also chosen to match the approach used in reference 13 for opaque large area roofing samples. As in that study reference reflectance standards are needed. An understanding of accuracy in reflectance thus requires consideration of the shadow impact on data from the two standards. Both diffuse back-scattering from transmitting samples and the black and white opaque reference standards used in the rig have in common that their signals come from the same area outside the shadow zone. Accuracy will thus be influenced by differences if any in reflectance spatial profiles. The related roof study [13] focused on arrays of tiles, which were diffuse reflectors. Sufficiently accurate results arise here in the same way for reflectance off diffuse transmitters when backscattering is also mainly diffuse, as comparisons later with spectrophotometer-integrating sphere data on a pigment doped transmitting sample confirms. Errors from shadowing effects will thus become important if the relative contribution of specular reflectance is large but the same diffuse standards with the same sensor orientation are used. This means in contrast to transmittance a modification of the technique will be needed before it could be considered as an accurate universal standard test for reflectance. Options for future study might include tilting the sample plane slightly to add any specular component, or using two specular and two diffuse standards, and possibly one with a mix of both types. However many skylight materials have low solar reflectance values as here. Rig values are under $10 \%$ with the pigmented case an exception, but it is highly diffuse, hence found to be accurate against spectrophotometer data. Data is confined here to samples which visibly are predominantly diffuse though one or two have a moderate specular reflectance component off one side. Thus for many complex glazings reflectance data in this simple set up has value. This experiment also indicates what sample types can yield good $R$ data and what may be needed for a wider range of reflectance profiles.

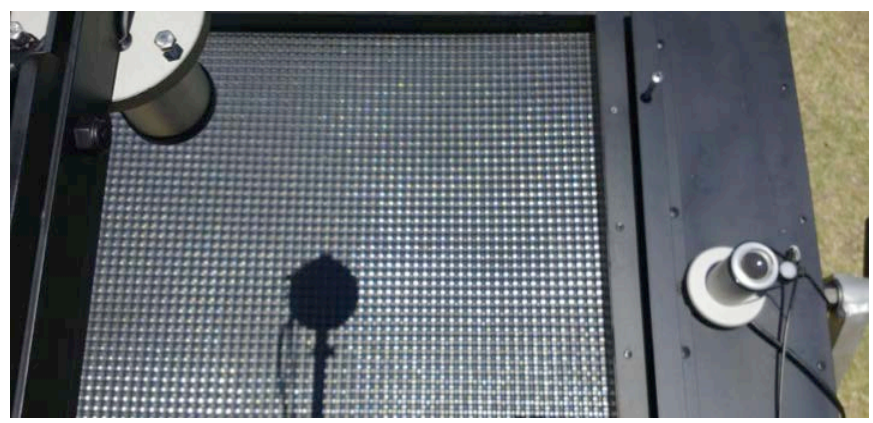

Fig. 7. The solar and daylight sensors mounted alongside a sample off which reflected radiation is being detected by a sensor pair. The sensor and support arm shadow is visible on the sample.

Transmittance in contrast has universal applicability if the secondary diffuser has the desired properties. As an initial quality test the transmittance properties of the standard diffuser were tested with no sample in place and matched values found for the same material using the spectrophotometer. Thus any specular large area transmitter could in principle be measured using its reduction of the solar or lux signal from the diffuser alone. However for most specular transmitting window products both $\mathrm{R}$ and $\mathrm{T}$ data is available. If not they are more easily found with a spectrophotometer, while this approach is aimed at complex transmitters unsuited to measuring with small integrating spheres. This system might however be of interest for some specular systems. For example it could be adapted to mount and check calculated $\mathrm{T}$ values for some double or triple glazings before and after adding complex internal features between panes.

The transmitting aperture is $580 \mathrm{~mm}$ square though sample sizes are larger typically 650 × $650 \mathrm{~mm}$ to allow simple mounting and locking in place, and sample inter-change, within the raised support structures seen on all sides in fig.7 around the aperture. Since we require one additional standardized sheet for 
transmittance and two for reflectance, and sample side alters when detection modes are changed, these raised sample support structures at the edges of the aperture are duplicated on both sides of the main panel. To assess the versatility of this rig as size varies a brief study of smaller samples with similar diffuser material as used in one large flat sample and some curvature was made. An example is in fig. 8 in transmittance mode. These can be mounted in custom support structures whose thickness enables mounting as for the flat panels (wood laminate was used in fig.8). Transmittance data despite the size contraction was close to what was expected for the sheet used from large flat panel data. An additional obvious source of reflectance error arose, namely a partial focusing effect linked to sample curvature. For R-data on any small area, very diffuse products with minimal focusing the role of larger relative size of detector shadow to sample area needs examination. Again though standards will scatter from the same non-shadowed areas. We concluded that for transmittance only this approach could form the basis of a universal single technique for curved panels.

The contribution to measured signal from surfaces other than the sample area within the detector's hemispherical field of view adds to sample data. They are accounted for within the

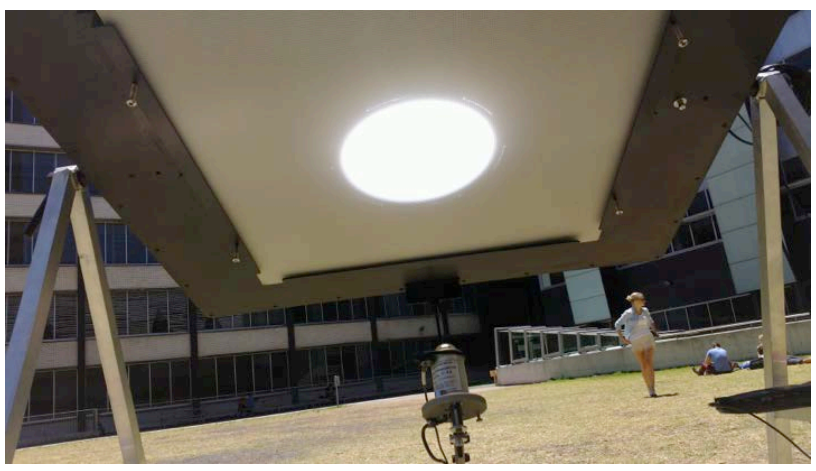

Fig. 8. Transmittance measurement set-up for a smaller curved sample mounted in a wooden frame and standard diffuser panel also in place to test geometric limitations.

measurement and data analysis protocol in different ways for $\mathrm{T}$ and $\mathrm{R}$ to eliminate their impact. This step is outlined in section 4 . Among background options for $\mathrm{R}$ is having the transmitted light pass through to the ground behind which could be a black cloth, or having a standard very black panel mounted immediately behind the sample. The latter is what was done in this study. Background signal can change with the former option but with a black backing plate its small impact is reliably quantified. As background signal adds to sample signal it can, if excessive, impact on accuracy. Thus steps are taken to minimize its contribution. The largest non-sample materials in the field of view are the sample mount and the detector support structure. Both are coated with a matt black paint, which yields spectrally flat surfaces across the whole solar range. This paint gave solar and visible hemispherical reflectance of $4.4 \%$ and $7.2 \%$ respectively. Such low numbers cause a useful reduction in background signal. Its total is still measured and explicitly included in the final analysis.
Shadowing from the normal detector mount rules out accurate reflectance from materials with a considerable specular component. Highly diffuse samples with low R, even with hot spots, cause little shadowing error due to our large sample area detector offset, and diffuse standards. Most complex materials of interest in skylight work have significant diffuse components and low reflectance values.

\section{Data collection and its analysis}

Transmittance data presented here used one homogenizer panel whose actual $\mathrm{T}$ value is not needed in the final calculations. Reflectance data used two standard reference panels, one white and one black, whose $\mathrm{R}$ values are required. The transmittance homogenizer had a pre-determined absolute value but this number was used only for reference and as a guide in its choice among other options. It is not used in the final calculations as it gives in effect the $\mathrm{T}=100 \%$ reference signal. Its requirements were a highly diffuse output combined with a high transmittance, for most incidence angles. Such a combination was rare until recently. The sample used had AM1.5 hemispherical Tsol $=0.88$ and Tvis $=0.89$ as measured with a spectrophotometer and $150 \mathrm{~mm}$ integrating sphere, then calculated using the AM1.5 spectrum. Its output when used outdoors was always quite uniform at a moderate distance from its surface. Alternative standard options were possible including acrylic specially doped with microspheres whose refractive index was close to but slightly different to that of the matrix [14].

The two reflectance standards were both diffuse. White and black-coated metal sheet is used with the white sheet having an AM1.5 hemispherical Rsol value of 0.82 , and Rvis value of 0.62 as found with the photopic eye spectrum. The black sheet had AM1.5 Rsol value of 0.044 and Rvis value of 0.072 .

\subsection{Transmittance measurement and calculation procedures}

Once the rig has all detector axes aligned to the solar beam direction for maximum signal the direct solar irradiance $\Phi$ sol $\left(\mathrm{Wm}^{-2}\right)$ and direct illuminance $\Phi$ vis (Lux) signals are recorded with the outer pyranometer and attached lux meter respectively. All subsequent irradiance and lux transmittance readings with the other two sensors (Xt,sol, Xt,vis) are scaled to these two values respectively in our methodology and the results are called Solar Transmittance Ratio (St) and Light Transmittance Ratio (Lt) so St $=[\mathrm{Xt}, \mathrm{sol} / \Phi$ sol $]$ and Lt $=[\mathrm{Xt}$,vis/Фvis $]$. ssol and $\Phi$ vis are re-recorded at least every time a new sample is mounted or after sufficient time has elapsed that slight shifts in solar intensity and shadow alignment are apparent, to ensure rig alignment is correct. In addition the small background signal from any indirect radiation $\mathrm{Xb}$,sol, $\mathrm{Xb}$,vis falling on the transmission pyranometer and lux sensor, mainly from the rig itself, and ground plus other surrounds of the rig, are recorded with the sample aperture blocked. This contribution is subtracted from all readings used in estimating $T$. It is not necessary in this procedure to monitor the signal with an open aperture as it is not needed in the calculations though it may be of interest to do so occasionally as along with the absolute direct measures of solar intensity and daylight lux it can give a strong indication of the degree of specularity in the solar radiation. Little diffuse component was apparent in this aperture data, as seen from its close proximity to the direct reading of Фsol and Фvis. 
The next pair of readings are after the full size homogenizer panel is mounted at the rear of the sample on the side nearest to the transmittance detector. It can be left in place for a sequence of different samples. Data with this reference standard is then recorded and the solar and light ratios calculated to yield quantities St(100) and Lt(100). They are labeled this way as after the small background correction they become the 100\% reading reference ratios. That is we wish to quantify all radiation falling on this panel, which is easily done from its actual transmittance ratio. It is useful to note that this homogenizer was found to yield the same value of the ratio $\mathrm{Xt}$,vis/Xt,sol as the direct solar ratio Фvis/Фsol. This implies that Tvis/Tsol 1.0 for the homogenizer panel which requires a near spectrally flat response across the solar spectrum. The ratio $\mathrm{Xt}$, vis/ $\mathrm{Xt}$,sol which is immediate, is useful as a measure of "light coolness" in units Lumens/Watt (L/W). For the rig results and those from the laboratory spectrophotometer, the homogenizer yields the same "light coolness" index as direct sunlight. This also means that simultaneous solar and visible sensor readings with sample and homogenizer both in place immediately yield the "light coolness" ratio factor of the light and solar energy transmitted by that sample without further analysis.

Finally the sample of interest is mounted on the front side of the sample holder and the homogenized light intensity reaching the detectors after passing through both it and the reference sheet are recorded. As before ratios are taken to yield St, Vt for each sample, while Sb,sol and Lb,vis denote the background ratios of type $\mathrm{Xb} / \Phi$. As a first approximation this recorded set of data is applied to calculate Tsol and Tvis as follows in equations (1) and (2).

$$
\begin{aligned}
& T \text { sol }=\frac{S t, \text { sol }-S b, \text { sol }}{S t(100)-S b, \text { sol }} \\
& T \text { vis }=\frac{L t, v i s-L b, v i s}{L t(100)-L b, v i s}
\end{aligned}
$$

A slightly more accurate result follows if account is taken of back reflection from the secondary or standard diffusing panel. Due to multiple reflection between panels apparent transmittance as given by equations (1) and (2) is a little higher than the true value. The reduction factor needed to find the actual result is given by the denominator in equation (3) which yields the final transmittance result.

$$
T_{\text {sol,corr }}=\frac{\text { Tsol }}{[1+R 1, \text { sol } * R 2, \text { sol }]}
$$

An analogous correction applies to Tvis. $R_{1, \text { sol }}$ and $R_{2, \text { sol }}$ are the solar reflectance of the two facing surfaces, and higher order multiple reflectance terms are omitted as they are of no practical significance. Panel orientation when mounting is an important consideration for many panels, as the correct $R$ values are needed in equation (3), while many of the samples for which this technique is needed have different surface structures on opposite sides, hence different $R$ values. In all the samples reported in this paper the largest correction from equation 3 was a lowering of the $\mathrm{T}$ value by $1.0 \%$. The worst possible case theoretically is not of practical interest in this work but would mean the results from (1) or (2) were high by a factor 1.026 or $2.6 \%$.

For the three samples which were most suited to testing with the existing standard aligned sphere method a comparison will be shown in the results section following between the transmittance values from this large panel procedure, and those using a $150 \mathrm{~mm}$ integrating sphere and spectrophotometer. Results either match or are more accurate with the new rig. The better rig accuracy was interesting and unexpected. It arose for one sample, which though casting a uniform diffuse transmitted beam on a distant screen when uniformly illuminated, had surface contours for which the spectrophotometer beam size was not large enough to yield a correct full surface average. That is spectrophotometer accuracy would need multiple beam positions and readings, or an enlarged beam. The sphere method of Nilsson et al [9], which is in essence a hybrid of the two approaches for samples without severe hot spots as in these three, might avoid this problem. Since transmittance is the main quantity of interest for both lighting supply and heat management from skylights and roof glazing, its accuracy is important. The homogenizer panel aims to produce the same output light patterns, even when the panel of interest displays varied density and spatial distribution of hot spots, by using 570 x $570 \mathrm{~mm}$ panels. The separation of sample and homogenizer panels of $25 \mathrm{~mm}$ enhances its ability to produce a constant output pattern. Thus this technique has promise for transmittance studies on complex glazings for both visible and solar radiation and may be a basis for a standard.

\subsection{Reflectance measurement and calculation procedures}

Reflectance is less straightforward and needs further technique development for some samples, but all initial results for flat panels are recorded here for use in (3), and for future reference. Some are accurate. Sample character is the main issue. Unlike transmittance, which is referenced to a $100 \%$ signal, reflectance relies on diffuse reference standards, one with low $\mathrm{R}$ and one with high $R$. As is well known with integrating spheres, unless the spectral response of standards especially the high $\mathrm{R}$ ones, are uniform across the range of interest errors can occur for some samples. Detector issues as in figure 7, include shadowing by the solar detector. A slight angle of incidence of order $8^{\circ}$ is of course the standard approach to approximating normal incidence in spectrophotometers and may finally be needed here as well. One initial aim was to explore how important reflectance shadowing was for diffuse transmittance samples referenced to two diffuse standards. Then shadowing has little or no final influence as it is proportionally common to all three, and also weak. The same standards cannot be used with this set up if sample specular reflectance is significant. When panel material absorptance is known it can be used when combined with transmittance data, to estimate the reflectance if rig $R$ values are uncertain due to specularity. One diffuse sample again had problems in the spectrophotometer due to sampling errors resulting from the small beam size.

The standard black and white reference panels are first mounted facing both the sun and the reflectance detectors as in 
figs. 7 and 8. Four solar and visible reflectance ratios are calculated and recorded from appropriate data. Replacing " $\mathrm{t}$ " with " $r$ " in the notation used above this data yields the ratios Sr(white), Lr(white), Sr(black), Lr(black). A better spectrally flat white reference is possible as used in some integrating spheres. It would however be very expensive over this large area and less durable in such a rig, as it has to be moved in and out.

The black standard sheet has a second use in this study. It is mounted in the rear holder with the sample of interest in front. It is intended to block any background signal from behind the rig and with Rsol $=0.044$ and Rvis $=0.072$ multiple reflections from it are almost, but not quite eliminated. Its aim was to ensure that the net reflected signal is dominated by the sheet itself. An alternative with no black panel behind the sample is possible as noted above. The relatively weak background signal from the detector field of view beyond the sample aperture is simply accounted for directly using a calibration plot based on our reference Rsol values of 0.82 and 0.04 and Rvis values of 0.62 and 0.072 . The slope and intercept values of (linear) plots of these known values against the ratios $\mathrm{Sr}$ and $\mathrm{Lr}$ given by the outdoor rig are used in a spread sheet to quickly find the value of any sample from its raw data. The slope $K$ and intercept $I$ are in this case used simply as in equation (4) for solar properties with Ksol and Isol defined in (5) and (6) respectively. A dash is added to Rsol in (4) to denote that this relation assumes the black back plate gives negligible contribution (corrected below). The equivalent relations for visible reflectance are by replacing "sol" with "vis".

$$
\begin{array}{r}
\text { Rsol }^{\prime}=\text { Ksol } \times S r, \text { sol } \\
+ \text { Isol }
\end{array}
$$

$K s o l=\frac{R \operatorname{sol}(\text { white })-R \operatorname{sol}(\text { black })}{S r, \operatorname{sol}(\text { white })-S r, \text { sol }(\text { black })}$

$I s o l=R \operatorname{sol}($ white $)-K s o l * S r, \operatorname{sol}($ white $)$

$$
\begin{array}{r}
\text { Isol }=\operatorname{Rsol}(\text { black })-\text { Ksol } \\
\times S r, \text { sol }(\text { black })
\end{array}
$$

A constant background signal impacts via a vertical shift in the calibration curve and is given quantitatively by the calibration intercept Isol given in equations (6). It is present in all cases. If solar intensity varies by a large amount during a test, recalibration is needed but our conditions were always similar even on different days, so the calibration curve barely changed. However it should definitely be recalculated for measurements on different days by re-running the black and white samples, which takes little time.

The results from equation (4) are not quite correct. They assume the back plate absorbs all incident radiation. For high transmittance samples low black reflectance can still add significant signal. This relative signal strength for low reflectance hence low sample signal is worse when transmittance is high. A correction is thus added for any signal arising from reflection off the black back. Incorporating the back-plate correction yields the final results for Rsol as

$$
R s o l=R s o l^{\prime}-(T s o l)^{2} \times R s o l(b l a c k)
$$

One sample had transmittance asymmetry of $10 \%$ depending whether surface features are up or down. If present such asymmetry can be accounted for in equation (7) by replacing (Tsol) ${ }^{2}$ by Tsol(up)Tsol(down). Reflectance results for curved samples were complicated by partial focusing in reflectance when one surface is smooth and for now only $\mathrm{T}$ values are relaible in these systems. Tsol using this rig and the methods of section 4.1 does give accurate results for the two curved surfaces sampled to date. If Tsol is available, and solar absorptance Asol is known then one can make an approximate estimate of reflectance. Curved material checked in the spectophotometer had a Tsol value close to that from the rig data. Asol is also of interest for modeling of SHGC. Among the following samples Asol ranged from $22 \%$ for the pigmented PMMA sheet to $2 \%$ for the thin, very clear polymer sheet with surface dimples. When structured polymer surfaces are present on at least one side extra internal reflection and hence absorption has been observed. For large area flat sheet their impacts are included but with small samples a significant fraction of trapped light escapes at the sample edges as often occurs [14]. In that situation a secondary pre-sphere diffuser [9] may not help.

\section{Results and discussion}

Data obtained on a range of complex polymer glazings with this new set-up has been processed using equations (1-7), while "coolness" ratio is found directly from the two sensor outputs. Final transmittance results and coolness of transmitted light appear in table 1, while reflectance results are in table 2. Also shown in each table where appropriate are the data obtained on suitable samples using spectrophotometer and integrating sphere for comparison. For three samples rig data is compared with spectrophotometer/150 mm sphere results using AM 1.5 for solar reflectance. Sphere data is less accurate for the dimpled PMMA due to its beam size relative to dimple size and spacing.

To the two significant figures used in table 1 the multiple reflection corrections of equation (3) had no impact except for a shift down of $1 \%$ for three data points which occurred in the data associated with the highest reflectance values (see table 2) of $45 \%, 62 \%$ and $14 \%$. Transmittance results for the top three samples agreed well with standard instrumentation except for the Tsol data for the two dimpled samples. A subsequent investigation with the spectrophotometer showed that moving its beam slightly on these samples gave significant shifts in Tsol. Closer inspection shows that its small beam size was unable to properly sample average response. Smooth regions between dimples reflected more strongly than raised areas and the beam position for the data above had been biased to the flat zone so $\mathrm{T}$ was underestimated. It thus appears that for these types of samples the new large area sample rig gives superior Tsol results to the small integrating sphere unless the latter uses a large beam size. It also seems from the visible data that this issue is more important in the NIR. The dimpled side being up or down makes little difference but slight asymmetry occurs in Tsol for 
the more accurate rig system. This initial analysis indicates that for the large rig transmittance errors are generally less than $1 \%$.

Triple wall polycarbonate in these tables has clear walls with vertical and tilted walls oriented primarily in the extrusion flow direction. Wall joints and variations in orientation lead to scattering and multiple internal reflections, which add to net reflectance (see table 2) and to absorptance. Orientation of wall structure seen in figure 3 has a small influence only on solar transmittance values, presumably because the near infra-red is where wall absorptance is strongest and Asol is then enhanced by more multiple reflection. Reflectance asymmetry for the four samples with one smooth surface is more obvious in table 2 for visible radiation. Because visible radiation is more weakly absorbed by polycarbonate than NIR solar radiation this may still have weak impact on Asol. For three samples in table 2 rig $R$ data is compared with spectrophotometer data.

The one significant discrepancy in table 2 among the three samples initially considered suited to both techniques is between the rig and laboratory data for visible reflectance from the high transmittance dimpled surface with dimples up and down. We believe in this case both techniques are in error but for opposite orientations. The sphere data is too low for dimples up and the rig data too low for dimples down. This sphere error arises as before from the small beam size sampling problems which means average diffuse reflectance with dimples up is low. The rig value of $\mathrm{R}=3.2 \%$ with dimples down is too low and exemplifies problems with specularity as when light strikes the smooth surface first reflected light is predominantly specular. High specularity means the apparent reflectance signal is artificially low because much of it should have come from the shadow zone formed by the specular solar beam and the detector which is here aligned to sample normal hence beam direction as seen in figs. 5 and 7. Further development is needed for reflectance including study of the offset of sample normal to the solar beam and use of specular and diffuse standards.

\section{Conclusion}

A new technique has been investigated aimed at directly and simply measuring solar and visible transmittance and reflectance on a range of complex polymeric glazing panels. Integrating spheres with similar capability would require mega-sizes of order 4 to 5 meters in diameter as large sample areas are needed. Most laboratories would be unable to accommodate such sizes. Initial tests gave promising results, especially for transmittance which is accurate because of the addition of a special homogenizing panel which transmits most incident radiation and diffuses it uniformly in the process.

For uniformly diffuse samples good agreement was generally found between this technique and using a spectrophotometer with a $150 \mathrm{~mm}$ integrating sphere. The only exceptions for transmittance involved problems with the standard sphere data for certain sample structures, not the new rig. Small beam sizes in the spectrophotometer did not correctly yield average properties in such samples. This new approach to transmittance appears thus to generally yield accurate results.

Table 1. Transmittance data obtained on the new rig for a range of optically complex polymer materials

\begin{tabular}{|c|c|c|c|c|c|}
\hline Sample & $\begin{array}{c}\text { "Coolness" } \\
\text { transmitted } \\
\text { light } \\
\text { (Lum./Watt) }\end{array}$ & $\begin{array}{c}\text { Transmittance } \\
\text { Tsol \% }\end{array}$ & $\begin{array}{c}\text { Solar } \\
\text { Transmittance } \\
\text { Tvis \% }\end{array}$ & $\begin{array}{c}\text { Int. } \\
\text { Sphere } \\
\text { Tsol \% }\end{array}$ & $\begin{array}{c}\text { Int. } \\
\text { Tphere } \\
\text { Tvis }\end{array}$ \\
\hline White pigmented 3 mm PMMA & 92 & 32 & 26 & 32 & 28 \\
\hline $\begin{array}{c}3 \text { mm PMMA, one surface } \\
\text { dimpled (contours down) }\end{array}$ & 108 & 90 & 92 & 84 & 92 \\
\hline $\begin{array}{c}3 \text { mm PMMA, one surface } \\
\text { dimpled (contours up) }\end{array}$ & 107 & 89 & 92 & 84 & 92 \\
\hline $\begin{array}{c}\text { Clear Triple Wall polycarbonate } \\
\text { (lines north-south) }\end{array}$ & 106 & 70 & 70 & & \\
\hline $\begin{array}{c}\text { Clear Triple Wall polycarbonate } \\
\text { (lines east-west) }\end{array}$ & 101 & 73 & 69 & & \\
\hline $\begin{array}{c}\text { PMMA inverted 1 cm square } \\
\text { prisms up-flat side down }\end{array}$ & 119 & 86 & 87 & & \\
\hline $\begin{array}{c}\text { PMMA inverted 1 cm square } \\
\text { prisms down- flat side up }\end{array}$ & 112 & 76 & 75 & & \\
\hline
\end{tabular}


Table 2. Reflectance data for the optically complex polymer materials in table 1 .

\begin{tabular}{|c|c|c|c|c|}
\hline Sample & $\begin{array}{c}\text { Solar } \\
\text { Reflectance } \\
\text { Rsol \% }\end{array}$ & $\begin{array}{c}\text { Visible } \\
\text { Reflectance } \\
\text { Rvis \% }\end{array}$ & $\begin{array}{c}\text { Int. } \\
\text { Sphere } \\
\text { Rsol \% }\end{array}$ & $\begin{array}{c}\text { Int. } \\
\text { Sphere } \\
\text { Rvis \% }\end{array}$ \\
\hline $\begin{array}{c}\text { White pigmented } 3 \text { mm PMMA, both } \\
\text { surfaces smooth }\end{array}$ & 45 & 62 & 47 & 62 \\
\hline $\begin{array}{c}3 \mathrm{~mm} \text { PMMA, dimpled surface up, } \\
\text { smooth down }\end{array}$ & 9.7 & 8.8 & 7.5 & 7.9 \\
\hline $\begin{array}{l}3 \text { mm PMMA, dimpled surface down, } \\
\text { smooth up }\end{array}$ & 6.5 & 3.2 & 6.7 & 7.1 \\
\hline $\begin{array}{l}\text { Clear Triple Wall polycarbonate } \\
\text { (lines north-south) }\end{array}$ & 9.7 & 8.0 & & \\
\hline $\begin{array}{c}\text { Clear Triple Wall polycarbonate } \\
\text { (lines east-west) }\end{array}$ & 8.9 & 14 & & \\
\hline $\begin{array}{l}\text { PMMA inverted } 1 \mathrm{~cm} \text { square prisms } \\
\text { (prisms up- flat side down) }\end{array}$ & 0.7 & 3.6 & & \\
\hline $\begin{array}{l}\text { PMMA inverted } 1 \mathrm{~cm} \text { square prisms } \\
\text { (prisms down- flat side up) }\end{array}$ & 3.8 & 8.3 & & \\
\hline
\end{tabular}

Reflectance though accurate for diffuse samples with low specular content requires development to be more widely applicable. The data from this instrument for the range of materials studied so far gives reliable output when used in the

\section{Acknowledgements}

The development of this apparatus, and our initial testing was supported by the Australian Skylight Industry Association (SIA) and The Australian Fenestration Rating Council (AFRC). We thank Steve Lynch of Skydome and Ian Murphy of Regent Skylights for much useful discussion, and Dr. Peter Lyons and Ian Bennie for useful comments, including guidance as to what

\section{References}

1. G.B. Smith and C. G Granqvist, "Green Nanotechnology: Solutions for sustainability and energy in the built environment" CRC press (Taylor and Francis), Boca Raton, USA, September, 2010 ISBN 978-1-4200-8532-7

2. H. Akbari, S. Menon and A. Rosenfeld, Global cooling: Increasing world wide urban albedo to offset $\mathrm{CO}_{2}$, Climate Change, 94 (200) 275 - 286.

3. I. Edmonds and G. B. Smith, Surface reflectance and conversion efficiency dependence of technologies for mitigating global warming, Renewable Energy, 36 (2011) 1343-1351.

4. ASTM (1996) E903-96 Standard test method for solar absorptance, reflectance and transmittance of materials using integrating spheres. ASTM, West Conshohocken PA.

5. ASTM (2006) E1918-06 Standard Test Method for Measuring Solar Reflectance of Horizontal and Low-Sloped Surfaces in the Field

6. ASTM (2013) E972-96 Standard Test Method for Solar Photometric Transmittance of Sheet Materials Using Sunlight.

7. ASTM (2009) E1084-86 Standard Test Method for Solar Transmittance (Terrestrial) of Sheet Materials Using Sunlight modeling of building thermal energy flows or lighting performance of skylights and roof glazing when these materials form part of the building or skylight structure. Transmittance dominates their energy control and lighting capabilities.

would be needed for a future standard. Regent Skylights contributed to the construction and design of the rig.

We are grateful to Dr. Helen Rose Wilson of the Institute of Solar Energy Systems in Freiburg, Germany (ISE) for her useful comments and valuable suggestions on aspects of this study.

8. The International Glazing Data Base, Lawrence Berkeley Laboratory http://windows.lbl.gov/g/IGDB/

9. A. M. Nilsson, A. Jonsson, J.C. Jonsson and A. Roos, Method for accurate transmittance measurements of low angle scattering samples using an integrating sphere with an entry port diffuser, Applied Optics, 50 (2011) 3981-3991.

10. WINDOW 7.1, 7.2 http://windows.lbl.gov/software/window/7/ 11. A. Gombert, C. Buhler, W. Hosfeld, J. Mick, B. Blaesi, G.Walze and P. Nitz, A rigorous study of diffraction effects on the transmission of linear dielectric micro-reflector arrays, Journal of Optics. A 6 (2004), 952-960.

12. D. Miller, J. Bengoechea, J. Bokria, M. Kohl, N. Powell, M. E. Smith, M. D. White, H. R. Wilson and J. H. Wohlgemuth, Proc. SPIE. 8825, Reliability of Photovoltaic Cells, Modules, Components, and Systems VI, 882509. (September, 2013) doi: $10.1117 / 12.2024372$

13. A. Akbari, R. Levinson and S. Stern, Procedure for measuring the solar reflectance of flat or curved roofing assemblies, Solar Energy 82 (2008) 648-655.

14. G.B. Smith, J. Jonsson and J. Franklin, Spectral global and diffuse properties of high performance translucent polymers for energy efficient lighting and skylights, Applied Optics, 42, 39813991, 2003. 